\title{
Incidencia de la personalidad y resiliencia en la aparición del burnout en una muestra de deportistas españoles
}

\section{Incidence of personality and resilience in the onset of burnout in a sample of Spanish athletes}

\author{
Ma Dolores García-Hernández ${ }^{1 *}$, Enrique Javier Garcés de los Fayos Ruiz \\ Juan González Hernández ${ }^{2}$ y Francisco José Ortín Montero ${ }^{1}$ \\ 1 Universidad de Murcia (España). \\ 2 Universidad de Granada (España).
}

\begin{abstract}
Resumen: El objetivo principal de esta investigación fue conocer los niveles de burnout en deportistas y la relación de este síndrome con la personalidad desde el Modelo de los Cinco Factores de Costa y McCrae, ańadiendo asimismo la resiliencia. La muestra estuvo constituida por 141 hombres y mujeres. Se utilizaron el IBD-R, NEO-FFI, Escala de Resistencia, e IPAQ para registrar y medir las variables anteriormente mencionadas. Los resultados apuntaron a que los participantes presentaban niveles moderados en todas las dimensiones del burnout. Además, ser hombre estaba asociado a mayores niveles de burnout, así como estar federado, participar en un deporte individual y pasar un mayor número de horas de entrenamiento. La edad no resultó ser una variable influyente en el padecimiento de este síndrome. Asimismo, los resultados indicaron un patrón de personalidad vulnerable del Modelo de los Cinco Factores, consistente en altos niveles de neuroticismo y bajos niveles de responsabilidad, amabilidad, apertura y extraversión. Por último, la resiliencia también fue una variable protectora del burnout. Palabras clave: emociones, ejercicio, rasgos, fortalezas, deporte
\end{abstract}

Abstract: The aim of this research was to know the level of burnout in athletes and the relation between this syndrome and personality from Costa and McCrae's five factors model, adding also the resilience. The sample consisted of 141 athletes $(M=21.20 ; S D=3.64)$. IBD-R, NEO-FFI, Resilience Scale, and IPAQ were used to record and measure the variables previously mentioned. Results indicated that the participants had moderated levels in all dimensions of burnout. Also, being a man was associated to higher burnout levels, as well as being federated, participating in an individual sport and spending a higher quantity of hours training. Age didn't turn out to be an influential variable in the suffering of this syndrome. Moreover, results indicated a vulnerable personality pattern from Five Factors Model, consisting on high levels of neuroticism and low levels of responsibility, kindness, openness and extraversion. Lastly, resilience was also a protective variable for burnout.

Keywords: emotions, exercise, traits, strengths, sport.

\section{Introducción}

Maslach y Jackson (1981, p.3) definieron el Burnout como "un síndrome tridimensional caracterizado por agotamiento emocional, despersonalización y reducida realización personal”. Esta, según Garcés de los Fayos (2004), es la definición aceptada en la actualidad por la mayoría de los investigadores del tema, siendo aplicable al contexto deportivo, tal y como afirman García-Parra, González y Garcés de los Fayos (2016). Asimismo, su definición está apoyada en la evidencia empírica del instrumento que estas mismas autoras desarrollaron: el Maslach Burnout Inventory (MBI) (Maslach y Jackson, 1981 y 1986).

Lo cierto es que el burnout sigue siendo una línea de investigación novedosa en el ámbito deportivo y que avanza a un ritmo demasiado lento, publicándose pocos trabajos (Garcés de los Fayos, Carlín y Carlín, 2010), siendo el estudio de este síndrome en deportistas escaso en comparación con el estudio del mismo en el contexto organizacional, donde surgió el término por primera vez con Freudenberger (1974), tal y

Dirección para correspondencia [Correspondence address]: $\mathrm{M}^{a}$ Dolores García-Hernández. E-mail: mariadolores.garcia43@um.es como muestran revisiones recientes como la de García-Parra et al. (2016).

Se considera por tanto de gran importancia ampliar el conocimiento sobre este tema, debido principalmente a la preocupación que causa el impacto psicológico en los deportistas que lo sufren. En este sentido, Garcés de los Fayos (2004) destacó que los deportistas, ante las presiones que sufren en el contexto deportivo, son una población de alto riesgo al mismo nivel que los trabajadores de servicios humanos, considerados tradicionalmente los de mayor riesgo de padecerlo, por lo que el síndrome es tan relevante en el contexto deportivo como en el organizacional. El coste fundamental para ellos es sobre todo motivacional y emocional, impidiéndole mantener la motivación que tenía inicialmente en su actividad, apareciendo un distanciamiento emocional de las personas relacionadas con esa actividad.

En la revisión bibliográfica realizada por García-Parra et al. (2016), los autores se refieren a las siguientes variables que influyen en la aparición o mantenimiento del síndrome: a) alto nivel de perfeccionismo desadaptativo y bajo nivel de perfeccionismo adaptativo, b) estrés, c) mayor tiempo de 
entrenamiento, d) experiencias negativas en el deporte, e) importancia que se le da al deporte, pasión obsesiva por el deporte, f) lesiones deportivas, edad (éxito temprano en el deporte), g) otros. En general, todos los estudios han señalado al estrés como un factor predictor del burnout (De Francisco, Arce, Del Pilar Vílchez y Vales, 2016).

No es de extrañar por tanto, que se encuentren cifras elevadas de incidencia de burnout en deportistas. Así, Rotella, Hanson y Coop (1991) cifran en 35\% la incidencia del síndrome; Weinberg y Gould (1995) lo sitúan en el 47\%; Jiménez, Jara y García (1995) en un 6\%; y Medina Mojena (2001) en una incidencia superior al 10\%. Más recientemente, De Francisco, Garcés de los Fayos y Arce (2013) situaron la prevalencia del burnout en deportistas en un $4 \%$, a lo que, si se le suman los deportistas con un alto riesgo de padecer el síndrome, se situaría el porcentaje en un $15 \%$.

Entre las consecuencias del síndrome del burnout, destacan las descritas por Gould, Tuffey, Udry y Loehr (1996): a) problemas físicos (enfermedades y lesiones), b) insatisfacción con estilo de vida y rol asociado al deporte, c) expectativas incumplidas, d) sentimientos de aislamiento, e) problemas de concentración, f) componentes afectivos negativos y, g) abandono. Asimismo, De Francisco et al. (2016) describen cómo pueden afectar a la totalidad de la persona, señalando síntomas físicos (dolor de cabeza, insomnio, fatiga, ausencia de apetito), comportamentales (bajo rendimiento, conducta rígida) y psicológicos (depresión, dificultad en las relaciones con los demás, pensamientos negativos, etc.).

No obstante, es importante señalar que la aparición de tales consecuencias estará en función de una compleja interacción entre las variables predictoras, su intensidad y frecuencia, así como la percepción de las mismas que tiene el deportista y su personalidad. Además, encontramos ciertas variables protectoras que también intervienen en esta relación.

Por otra parte, cada vez hay una mayor evidencia empírica de la relación entre la personalidad y el burnout (Alarcon, Eschleman y Bowling, 2009). En relación con esto, la personalidad influye en la manera de interpretar y reaccionar ante las circunstancias y la forma de afrontar el estrés (Bermúdez, 1999), por lo que no es de extrańar que pueda tener gran importancia en la vulnerabilidad de padecer este síndrome.

El enfoque de la personalidad que se va a adoptar en este estudio es el Modelo de los Cinco Factores, que clasifica los rasgos de personalidad en cinco dimensiones: extraversión, amabilidad, responsabilidad, neuroticismo y apertura a la experiencia (Costa y McCrae, 2008).

Con respecto a la relación entre la personalidad y el burnout, cabe destacar que entre las variables que pueden provocar la aparición del síndrome encontramos las características de la personalidad señaladas por Garcés de los Fayos (1999): a) inestabilidad emocional, b) menor entusiasmo, c) poco control de la ansiedad, d) sentimientos de culpabilidad, e) alexitimia, f) ser reservado y, g) reprimido. Asimismo, se ha encontrado que las personas con personalidad tipo A tienen más probabilidad de padecer este síndrome (Garcés de los Fayos y Cantón, 1995). Por último, el perfeccionismo se ha relacionado constantemente con el burnout en los deportistas (Hill, Hall, Appleton, y Kozub, 2008).

Esta evidencia coincide con lo señalado en el estudio de Kania, Meyer y Enersole (2009), al indicar que la varianza explicada por los factores de personalidad ante la aparición del burnout es más del doble frente a los ambientales en las tres dimensiones del síndrome.

Asimismo, entre las variables protectoras mencionadas anteriormente encontramos que las personas con una personalidad resistente están más protegidas de padecer burnout. Esta personalidad resistente fue descrita por Garcés de los Fayos (1999, p. 50) de la siguiente manera: "un sentimiento de compromiso hacia sí mismo y el trabajo, una percepción del control del ambiente, así como una actitud a acercarse a los cambios de la vida con una actitud de desafío antes que de amenaza".

Sin embargo, no se han encontrado investigaciones que estudien la relación entre los niveles de burnout y las dimensiones de personalidad desde el Modelo de los Cinco Factores de Costa y McCrae (2008), lo cual es uno de los objetivos del presente estudio.

Por otra parte, es bien conocido que la vida de los deportistas y su entorno deportivo está llena de factores estresantes (Arnold y Fletcher, 2012); y tales factores inciden en la probabilidad de padecer burnout por parte del deportista, ya que prácticamente todos los estudios realizados han señalado al estrés como un factor predictor del burnout (De Francisco et al., 2016). De esta manera, es importante estudiar una de las variables moderadoras y protectoras ante estas circunstancias: la resiliencia.

La resiliencia puede ser definida como una habilidad individual para adaptarse y responder al estrés y la adversidad (American Psychological Association, 2015). La escala más utilizada para evaluar este constructo es la Escala de Resiliencia de Wagnild y Young (1993). La adaptación española de esta escala fue llevada a cabo por Ruiz-Barquín et al. (2012), y evalúa el grado de resiliencia como una característica de la personalidad que favorece la adaptación (Wagnild y Young, 1993).

Este constructo ha sido estudiado en numerosos trabajos en el ámbito deportivo. Como ejemplo, podemos seńalar el estudio de Yi, Smith y Vitaliano (2005), en el que al estudiar a atletas resilientes y no resilientes, encontraron que los resilientes tenían más capacidad para afrontar los eventos negativos y estresantes de la vida.

Numerosos estudios coinciden en afirmar en diversos ámbitos que la resiliencia se asocia con menos probabilidad de sufrir desórdenes mentales, trastornos de conducta o bajo ren- 
dimiento (Rew, Taylor-Sheehafer, Thomas y Yockey, 2001). Concretamente, en el ámbito deportivo la resiliencia ha sido relacionada con la prevención de lesiones físicas en deportistas y la protección trastornos psicológicos (Currie y Johnston, 2016). Hosseini y Besharat (2010) también encontraron en su estudio que la resiliencia se asocia positivamente con el rendimiento deportivo y el bienestar psicológico, describiendo una relación negativa con los trastornos psicológicos.

En este sentido, es por tanto de esperar que la resiliencia se relacione de forma negativa con el burnout. Efectivamente, podemos encontrar varios estudios en los que se demuestra esto: algunas investigaciones cualitativas han mostrado que existe una relación entre resiliencia y burnout (Edward, 2005; Howard y Johnson, 2004). Asimismo, en 2014, Reche, Tutte y Ortín llevaron a cabo una investigación acerca de las variables resiliencia, optimismo y burnout, encontrando que la resiliencia está asociada a un bajo nivel de burnout.

\section{Objetivos}

Por todo lo explicado anteriormente, el objetivo de este estudio fue analizar el nivel de burnout en deportistas, con la pretensión de descubrir las diferencias existentes en función de la edad, sexo, y sus condiciones de práctica de actividad física regular. Se pretendió indagar además sobre un patrón de personalidad vulnerable o protector ante el burnout desde el Modelo de los Cinco Factores, teniendo en cuenta además la resiliencia.

Como hipótesis, se planteó que se encontrarían niveles moderados-bajos de burnout en la muestra, esperando que se presentase en mayor grado en mujeres, deportistas federados, aquellos que practicasen un deporte individual, más horas de deporte, y que tuviesen menor edad. Asimismo, en cuanto al patrón de personalidad asociado al burnout, se planteó como hipótesis que los deportistas con mayores niveles de amabilidad, extraversión, apertura y resiliencia presentarían menor grado de burnout, siendo mayor en los que puntuasen alto en neuroticismo y responsabilidad.

\section{Método}

\section{Muestra}

La muestra estuvo constituida por 141 deportistas (a partir de 18 años; $M=21.20 ; D T=3.64)$ que realizan actividad física de manera regular (ver Tabla 1). Como criterio de selección se estableció que, siguiendo las recomendaciones de la OMS sobre la actividad física en adultos (WHO, 2010), realizasen alguna actividad física intensa o moderada como mínimo 3 veces por semana. Finalmente, 70 de ellos realizaban un deporte individual (49.6\%) y 71 un deporte colectivo $(50.4 \%)$.

Tabla 1. Distribución de los participantes por intensidad de la actividad física y su frecuencia (días/semana), sexo y federado/no federado.

\begin{tabular}{|c|c|c|c|c|c|c|}
\hline & & Hombre & Mujer & Federado & No federado & Total \\
\hline \multirow{4}{*}{$\begin{array}{l}\text { Actividad física ALTA } \\
\text { (implican un esfuerzo intenso en } \\
\text { cuanto a tiempo de dedicación) } \\
\text { Total }\end{array}$} & Baja & $2(3.08 \%)$ & $2(2.63 \%)$ & $1(2.86 \%)$ & $3(2.83 \%)$ & 4 \\
\hline & Moderada & $23(36.92 \%)$ & $39(51.32 \%)$ & $10(28.57 \%)$ & $52(49.06 \%)$ & 62 \\
\hline & Vigorosa & $40(61.54 \%)$ & $35(46.05 \%)$ & $24(68.57 \%)$ & $51(48.11 \%)$ & 75 \\
\hline & & 65 & 76 & 35 & 106 & 141 \\
\hline \multirow{4}{*}{$\begin{array}{l}\text { Actividad física MODERADA } \\
\text { (no implican un gran esfuerzo en } \\
\text { cuanto a tiempo de dedicación) } \\
\text { Total }\end{array}$} & Baja & $16(24.62 \%)$ & $24(31.58 \%)$ & $9(25.71 \%)$ & $31(29.25 \%)$ & 40 \\
\hline & Moderada & $28(43.08 \%)$ & $32(42.11 \%)$ & $13(37.14 \%)$ & $47(44.34 \%)$ & 60 \\
\hline & Vigorosa & $21(32.31 \%)$ & $20(26.32 \%)$ & $13(37.14 \%)$ & $28(26.42 \%)$ & 41 \\
\hline & & 65 & 76 & 35 & 106 & 141 \\
\hline
\end{tabular}

\section{Instrumentos}

Se administraron los siguientes cuestionarios:

- Inventario de Burnout en Deportistas Revisado (IBDR) (Garcés de los Fayos, De Francisco y Arce, 2012): A lo largo de sus 19 items, el instrumento consta de tres dimensiones: agotamiento emocional (e.g., "Me encuentro al límite de mis posibilidades") ( $\alpha=.81)$, despersonalización (e.g., "Realmente no me importa lo que les ocurra a las personas que me rodean en mi actividad deportiva" $(\alpha=.78)$ y reducida realización personal (e.g., "Estoy emocionalmente defraudadola con el trabajo deportivo que realizo" ( $\alpha=.82)$. Respecto a su fiabilidad total, se obtuvo una consistencia interna buena para el presente estudio $(\alpha=.85)$.

- NEO-FFI (Costa y McCrae, 1992). Basado en el Modelo de los Cinco Factores, se trata de la versión reducida y adaptada al castellano (Aluja, Blanch, Sole, Dolcet y Gallart, 2009), y descrita en 60 items correspondientes a las dimensiones: extraversión (e.g., "Algunas personas piensan de mi que soy frio y calculador" $(\alpha=.84)$, amabilidad (e.g., "Disfruto mucho hablando con la gente" ( $\alpha$ 
$=.73$ ), responsabilidad (e.g., "Tengo unos objetivos claros y me esfuerzo por alcanzarlos de forma ordenada" $(\alpha=.83)$, neuroticismo (e.g., "A veces me parece que no valgo absolutamente nada") ( $\alpha=.87)$ y apertura a la experiencia (e.g., "A veces pierdo el interés cuando la gente habla de cuestiones muy abstractas y teóricas" $(\alpha=.82)$. Respecto a su fiabilidad total, se obtuvo una consistencia interna aceptable para el presente estudio $(\alpha=.71)$.

- Escala de Resiliencia de Wagnild y Young (1993). Concretamente, se utilizó la adaptación española de esta escala llevada a cabo por Ruiz-Barquín, De la VegaMarcos, Poveda, Rosado y Serpa (2012). Consta de 25 items distribuidos en dos factores: Competencia Personal (e.g., "Puedo afrontar problemas yo sólo si es necesario") ( $\alpha=.77)$ y Aceptación de uno mismo y de la vida (e.g., "Mi vida tiene sentido") ( $\alpha=$.66). Respecto a su fiabilidad, se obtuvo una consistencia interna adecuada para la presente muestra $(\alpha=.81)$.

- Cuestionario internacional de actividad física (IPAQ) en su formato corto autoadministrado de los últimos 7 días para adultos (International Physical Activity Questionnaire, 2017): se utilizó para conocer la actividad física realizada por los participantes. Para ello, incluía preguntas referidas a los días/semana y las horas/ día dedicados a realizar alguna actividad física intensa o moderada en los últimos 7 días. Además, se añadió una pregunta referida a si la actividad física realizada se lleva a cabo de forma individual o colectiva, y si estaban federados o no.

\section{Procedimiento}

Los cuestionarios fueron administrados en presencia de un investigador de forma grupal a los participantes, que accedieron voluntariamente a participar en la investigación tras la aplicación de un consentimiento informado que garantizaba la confidencialidad de sus datos. Se comenzó con una breve explicación del estudio. El tiempo de administración para completar todos los cuestionarios fue de aproximadamente 20 minutos en total.

\section{Análisis de datos}

El análisis de datos se llevó a cabo mediante el paquete estadístico IBM SPSS en su versión 21.0. Para constatar la consistencia interna de los instrumentos, se realizaron pruebas de fiabilidad (coeficiente alpha de Cronbach), para la valoración de la bondad del ajuste normalizado se aplica la prueba Kolmogorov-Smirnov a la población, con la valoración del uso de pruebas paramétricas. La distribución de la muestra se refleja a través de análisis descriptivos [frecuencias y medidas de tendencia central y dispersión (media, desviación típica, asimetría, curtosis y varianza)]. Con el propósito de comparar las variables objeto de estudio en cuanto a sexo, tipo de deporte (individual vs colectivo) y condición deportiva (federado vs no federado), se realizaron análisis diferenciales (pruebas T), y para la relación de las variables entre sí, tanto correlacional como predictiva, se realizaron análisis de correlación lineal (Pearson) y análisis de regresión lineal simple, respectivamente.

\section{Resultados}

En primer lugar, se realizó el análisis de los niveles de burnout de los participantes por dimensiones. Se obtuvo una puntuación media en la dimensión agotamiento emocional (AE) de $1.89(D T=.39$; varianza = .15; índice de asimetría de Fisher $\left(\mathrm{g}_{1}\right)=-.97$; curtosis $=2.72$ ). Concretamente, 19 participantes presentaban niveles bajos de esta dimensión (13.5\%), 118 participantes presentaban niveles moderados (83.7\%), y 4 participantes presentaban niveles altos $(2.8 \%)$. Respecto a la dimensión despersonalización (D), se obtuvo una puntuación media de $1.87(D T=.52$; varianza =.28; asimetría de Fisher $\left(\mathrm{g}_{1}\right)=-.16$; curtosis $\left.=.41\right)$. Un total de 30 participantes obtuvieron puntuaciones bajas (21.3\%), 100 presentaron puntuaciones moderadas (70.9\%), y 11 obtuvieron puntuaciones altas $(7.8 \%)$. Por último, respecto a la dimensión reducida realización personal (RRP), la puntuación media fue de $2.02\left(D T=.28\right.$; varianza $=.08$; asimetría de Fisher $\left(\mathrm{g}_{1}\right)=.76$; curtosis $=10.1)$. Concretamente, 4 participantes presentaron niveles bajos en esta dimensión $(2.8 \%)$; 130 , niveles moderados (92.2\%); y 7, niveles altos (5\%).

A continuación, se realizó un contraste de hipótesis sobre dos medias independientes (prueba T) para cada dimensión del burnout en relación con el sexo. Así, para la dimensión AE, se cumplió el supuesto de homogeneidad de varianzas según la prueba de Levene, no encontrándose diferencias estadísticamente significativas entre ambos sexos (hombres: $M=1.85 ; D T=.64 ;$ mujeres: $M=1.67 ; D T=.61 ; t(139)$ $=1.66 ; p>.05)$. Sin embargo, el tamaño del efecto encontrado fue de magnitud baja pero relevante según el criterio de Cohen $(d=.28)$. En cuanto a la dimensión $\mathrm{D}$, se cumplió el supuesto de homogeneidad de varianzas, encontrándose diferencias estadísticamente significativas entre los niveles de despersonalización de ambos sexos (hombres: $M=2.07 ; D T$ $=.77$; mujeres: $M=1.64 ; D T=.67 ; t(139)=3.53 ; p<.05)$. El tamaño del efecto fue de magnitud media-alta $(d=.6)$. Por último, respecto a la dimensión RRP, no se cumplió el supuesto de homogeneidad de varianzas, no encontrándose diferencias estadísticamente significativas entre ambos sexos (hombres: $M=2.04 ; D T=.53$; mujeres: $M=2.03 ; D T=.68$; $t(138)=.09 ; p>.05)$. El tamańo del efecto fue nulo $(d=.01)$.

También se realizó la prueba $\mathrm{T}$ para cada dimensión del burnout en relación con estar o no federado. Cabe señalar 
que se cumplió el supuesto de homogeneidad de varianzas en todos los casos que se muestran a continuación. En cuanto a la dimensión AE, no se encontraron diferencias estadísticamente significativas entre ambos grupos (federado: $M=1.85$; $D T=.75$; no federado: $M=1.72 ; D T=.59 ; t(139)=1.10 ; p>$ .05). Sin embargo, el tamaño del efecto fue de magnitud baja $(d=.29)$. Para la dimensión $\mathrm{D}$ tampoco se encontraron diferencias estadísticamente significativas (federado: $M=2.0$; $D T=.78$; no federado: $M=1.78 ; D T=.73 ; t(139)=1.50$; $p>.05)$. Sin embargo, el tamaño del efecto fue de magnitud baja $(d=.29)$. Finalmente, en la dimensión RRP tampoco se encontraron diferencias estadísticamente significativas (federado: $M=2.09, D T=.69$; no federado: $M=2.02, D T=.58$; $t(139)=.64 ; p>.05)$. El tamaño del efecto fue de magnitud nula $(\mathrm{d}=.1)$.

Asimismo, se realizó la prueba $T$ en relación a realizar un deporte individual o colectivo. Se cumplió el supuesto de homogeneidad de varianzas en todos los casos que se muestran a continuación. En este sentido, para la dimensión AE no se encontraron diferencias estadísticamente significativas entre los participantes que realizan un deporte individual o colectivo (individual: $M=1.85 ; D T=.65$; colectivo: $M=1.65, D T=$ $.6 ; t(139)=1.88 ; p>.05)$. Sin embargo, el tamańo del efecto fue de magnitud baja $(d=.32)$. En cuanto a la dimensión D, tampoco hubieron diferencias estadísticamente significativas entre ambos grupos (individual: $M=1.81, D T=.76$; colectivo: $M=1.86, D T=.73 ; t(139)=-.45 ; p>.05)$. El tamańo del efecto fue de magnitud nula $(\mathrm{d}=-.08)$. Por último, en cuanto a la dimensión RRP, sí se encontraron diferencias estadísticamente significativas entre ambos grupos (individual: $M=$ 2.18, $D T=.66$; colectivo: $M=1.89, D T=.53 ; t(139)=2.87$; $p<.05)$. Además, el tamaño del efecto fue de magnitud media $(d=.5)$.

El análisis de correlación lineal, señala el comportamiento de las variables objeto de estudio, en la muestra analizada (Tabla 2), en cuanto a su relación colineal $\left(\mathrm{r}_{\mathrm{xy}}\right)$. Destacando la relación positiva entre $\mathrm{AE}$ y neuroticismo, y las inversas con extraversión, amabilidad, responsabilidad, competencia personal y aceptación. De la misma manera, D muestra únicamente relaciones inversas con apertura, amabilidad, responsabilidad, competencia personal y aceptación. Y por último, RRP, señala relación positiva con neuroticismo, e inversas con extraversión, apertura, amabilidad, responsabilidad, competencia y aceptación. Además, pueden comprobarse como los rasgos de amabilidad, responsabilidad, y extraversión, se relacionan positivamente con los recursos resilientes, y estos a su vez de manera negativa con el neuroticismo. Asimismo, debe destacarse que no hubo ninguna relación significativa con la edad de los participantes de la muestra.

Tabla 2. Análisis correlacional. Coeficiente rxy entre los factores de personalidad, los factores de resiliencia y las dimensiones del burnout.

\begin{tabular}{|c|c|c|c|c|c|c|c|c|c|c|c|}
\hline $\mathrm{N}=141$ & K-S & 1 & 2 & 3 & 4 & 5 & 6 & 7 & 8 & 9 & 10 \\
\hline 1. Agotamiento Emocional & .20 & $(.81)$ & $.32^{* *}$ & $.44^{* *}$ & $.32^{* *}$ & $-.29^{* *}$ & -.07 & $-.23^{* *}$ & $-.19^{*}$ & $-.27^{* *}$ & $-.20^{*}$ \\
\hline 2. Despersonalización & .21 & & $(.78)$ & $.17^{*}$ & $.18^{*}$ & -.04 & $-.25^{* *}$ & $-.33^{* *}$ & $-.19^{*}$ & $-.20^{* *}$ & -.12 \\
\hline 3. Reducida Realización Personal & .18 & & & $(.82)$ & $.38^{* *}$ & $-.35^{* *}$ & $-.21^{*}$ & $-.21^{*}$ & $-.22^{* *}$ & $-.39^{* *}$ & $-.29^{* *}$ \\
\hline 4. Neuroticismo & .22 & & & & $(.87)$ & $-.35^{* *}$ & .03 & $-.37^{* *}$ & $-.37^{* *}$ & $-.50^{* *}$ & $-.61^{* *}$ \\
\hline 5. Extraversión & .19 & & & & & $(.84)$ & .09 & .14 & .10 & $.31^{* *}$ & $.22^{* *}$ \\
\hline 6. Apertura & .23 & & & & & & $(.82)$ & $.22^{* *}$ & -.07 & .16 & .12 \\
\hline 7. Amabilidad & .20 & & & & & & & $(.73)$ & $.23^{* *}$ & $.26^{* *}$ & $.34^{* *}$ \\
\hline 8. Responsabilidad & .19 & & & & & & & & $(.83)$ & $.55^{* *}$ & $.34^{* *}$ \\
\hline 9. Competencia personal & .22 & & & & & & & & & $(.77)$ & $.57^{* *}$ \\
\hline 10. Acept. de uno mismo y de la vida & .21 & & & & & & & & & & $(.81)$ \\
\hline
\end{tabular}

*. La correlación es significante al nivel .05 (bilateral).

**. La correlación es significativa al nivel .01 (bilateral).

El análisis de regresión lineal simple entre cada una de las variables que presentaron una correlación lineal significativa para observar si ese tiempo dedicado a la actividad física era un buen predictor de cada una de las dimensiones del burnout. De este modo, se encontró que las horas por día dedicadas 5 actividades físicas moderadas son un buen predictor del $\operatorname{AE}(F(1,139)=4.04 ; p<.05 ; \beta 1=.13)$. Sin embargo, el valor de $R 2$ corregido fue de .02 (magnitud nula). Por otra parte, se halló que las horas por día dedicadas a actividades físicas intensas eran un buen predictor de la $\mathrm{D}(F(1,139)=$ $8.63 ; p<.05 ; \beta 1=.23)$. Sin embargo, el valor de $R 2$ corregido fue de .05 .

Según estos resultados, se realizó un análisis de regresión lineal simple con cada una de las correlaciones significativas para observar qué variables de personalidad son buenos predictores de cada una de las dimensiones del burnout.

En cuanto al neuroticismo, el contraste de hipótesis con la prueba F de ANOVA nos condujo a considerar que resultó ser 
un buen predictor de las dimensiones $\mathrm{AE}[F(1,139)=15.32$; $p<.05 ; \beta 1=.28 ; R 2$ corregido $=0.12], \mathrm{D}[F(1,139)=4.55 p$ $<.05 ; \beta 1=0.29 ; R 2$ corregido $=.13]$, y RRP $\left[F_{(1,139)}=23.14\right.$; $p<.05 ; \beta 1=.32$; con un tamaño del efecto bajo-medio, $R 2$ corregido $=.14]$.

Asimismo, la extraversión resultó ser un buen predictor de las dimensiones $\mathrm{AE}[F(1,139)=13.14 ; p<.05 ; \beta 1=-.32 ; R 2$ corregido = .18] y RRP $[F(1,139)=19.58 ; p<.05 ; \beta 1=-.37$; con un tamańo del efecto bajo-medio, $\mathrm{R}^{2}$ corregido=.12].

Respecto a la apertura, resultó ser un buen predictor de las dimensiones $\mathrm{D}[F(1,139)=9.22 ; p<.05 ; \beta 1=-.30 ; R 2$ corregido $=.16]$ y $\operatorname{RRP}[F(1,139)=6.69 ; p<.05 ; \beta 1=-.21$; $R 2$ corregido $=.15]$.

La amabilidad también resultó ser un buen predictor de las dimensiones $\operatorname{AE}[F(1,139)=7.90 ; p<.05 ; \beta 1=-.29 ; R 2$ corregido $=.17], \mathrm{D}[F(1,139)=17.22 \mathrm{p}<.05 ; \beta 1=-.50 ; R 2$ corregido $=.19]$, y RRP $[F(1,139)=6.73 ; p<.05 ; \beta 1=-.264$; $R 2$ corregido $=.18]$.

Por último, respecto a la dimensión responsabilidad, resultó ser un buen predictor de las dimensiones $\mathrm{AE}[F(1,139)$ $=5.40 ; p<.05 ; \beta 1=-.21 ; R 2$ corregido = .13), $\mathrm{D}[F(1,139)=$ $5.07 ; p<.05 ; \beta 1=-.24 ; R 2$ corregido $=.13)$ y $\operatorname{RRP}[F(1,139)$ $=7.38 ; p<.05 ; \beta 1=-.24 ; R 2$ corregido $=.14)$.

Finalmente, el análisis de regresión lineal simple de cada dimensión de burnout con cada una de estas variables de resiliencia, encontró que la competencia personal era un buen predictor del $\mathrm{AE}[\mathrm{F}(1,139)=10.66 ; p<.05 ; \beta 1=-.27 ; R 2$ corregido $=.17]$, la $\mathrm{D}[F(1,139)=5.60 p<.05 ; \beta 1=-.37 ; R 2$ corregido=.13], y la RRP $[F(1,139)=25.59 ; p<.05 ; \beta 1=$ -.448; $R 2$ corregido $=.15)$. Asimismo, la aceptación de uno mismo y de la vida también resultó ser un buen predictor de las dimensiones $\operatorname{AE}[F(1,139)=5.64 ; p<.05 ; \beta 1=-.17 ; R 2$ corregido $=.13)$ y RRP $[F(1,139)=12.94 ; p<.05 ; \beta 1=-.250$; $R 2$ corregido=.18].

\section{Discusión}

El objetivo de la presente investigación consistía en conocer la relación del burnout con la personalidad desde el Modelo de los Cinco Factores, ańadiendo asimismo la resiliencia, en deportistas o personas que realizan actividad física de forma regular. Además, se pretendía descubrir si existen diferencias en los niveles de burnout en función del sexo, la edad, si la actividad física o deporte es individual o colectiva, si está federado o no, así como según el tiempo dedicado al mismo.

En primer lugar, se encontró una destacada mayor proporción de deportistas con niveles moderados de burnout en las tres dimensiones de este síndrome; seguidos de los participantes con niveles bajos, especialmente en las dimensiones agotamiento emocional y despersonalización. La dimensión que más padecen los deportistas, según los resultados obtenidos, es la reducida realización personal; difiriendo de estudios anteriores en los que se halló que la más padecida era la despersonalización (De Francisco et al., 2013).

Además, se pudo concluir que el sexo, estar o no federado, modalidad deportiva (individual o colectiva), y el tiempo de entrenamiento son variables relevantes en relación con el síndrome. Así, se encontró que los hombres presentaban mayores niveles de burnout, así como los federados y los participantes que realizaban un deporte individual. Asimismo, un mayor número de horas al día dedicadas a una actividad física moderada se asoció a mayores niveles de agotamiento emocional; encontrándose también mayores niveles de despersonalización asociados a la mayor cantidad de horas dedicadas a actividades físicas intensas. Sin embargo, no se puede afirmar que dicha relación sea predictiva.

Concretamente, en relación al sexo, se obtuvo que los niveles de agotamiento emocional y despersonalización eran mayores en hombres que en mujeres. Estos resultados difieren de los de otras investigaciones que afirman que el nivel burnout es mayor en mujeres, como las realizadas por De Francisco et al. (2013), Reynaga $(2008,2009)$ y Reynaga y Pando (2005). Sin embargo, nuestros resultados coinciden con los obtenidos por De Francisco et al. (2013) en las dimensiones despersonalización y reducida realización personal. Por lo tanto, parece que el sexo puede ser una variable relevante en relación con los niveles de burnout, aunque no hay resultados concluyentes ya que se siguen encontrando resultados diversos, tal y como muestra García-Parra et al. (2016) en su revisión.

Respecto a la influencia de estar federado o no en relación con el burnout, no se encontraron investigaciones previas que analizaran este aspecto, por lo que esta parece ser una aportación innovadora. Nuestros resultados, que mostraban mayores niveles de agotamiento emocional y despersonalización en los deportistas federados, se podrían explicar por las presiones deportivas que padecen, cuyos estresores podrían ser mayores.

Por otra parte, los mayores niveles de burnout obtenidos en deportes individuales (concretamente, en las dimensiones agotamiento emocional y reducida realización personal), coinciden con los resultados del estudio de Gustaffson, Kenttä, Hassmén y Lundqvist (2007). Consideramos que esto podría ser explicado por las exigencias psicológicas de las dinámicas de la modalidad individual, al no contar con el apoyo social de compañeros de equipo con los que afrontar las situaciones deportivas estresantes, etc. En este sentido, otra posible orientación para futuras investigaciones sería el registro de qué deporte o actividad física concreta realizan y observar si existen diferencias, ya que podría ser una variable influyente.

En cuanto a la influencia del tiempo de entrenamiento en los niveles de burnout, nuestros resultados coinciden con los presentados por García-Parra et al. (2016) en su revisión, ya 
que hay estudios que muestran que el volumen de entrenamiento se relaciona positivamente con el burnout.

Sin embargo, la edad no parece ser una variable influyente en la aparición del burnout, coincidiendo nuestros hallazgos con los de otros estudios como el de De Francisco et al. (2013), que tampoco encontró diferencias significativas en función de la edad en ninguna de las dimensiones del burnout. Podría ser, por tanto, que la edad no sea una variable relevante en relación con este síndrome, a pesar de esperarse que fuera mayor en deportistas de menor edad al contar presumiblemente con menores habilidades de afrontamiento.

Por otra parte, se halló un patrón de personalidad en deportistas desde el Modelo de los Cinco Factores asociado a mayores niveles de burnout; es decir, un patrón de personalidad vulnerable a este síndrome; constituyendo una importante aportación innovadora de este estudio ya que no se ha encontrado ninguno previo lo investigase concretamente desde este modelo. Este patrón consistiría en altos niveles de neuroticismo y bajos niveles de responsabilidad, amabilidad, apertura y extraversión. Un patrón de personalidad con niveles contrarios en cada dimensión protegería de padecerlo.

Por lo tanto, la personalidad es una variable moderadora e influyente en la aparición del burnout, pudiendo actuar como factor de riesgo o como variable protectora en función de sus características.

Por último, la resiliencia es otra variable de personalidad relacionada con este síndrome. Así, altos niveles de resiliencia se asocian a bajos niveles de burnout, por lo que es asimismo una variable protectora y moderadora ante los numerosos estresores asociados al ámbito deportivo. Estos resultados coin- ciden con los obtenidos en otras investigaciones como la de Reche et al. (2014).

Como posibles limitaciones de este estudio, cabe señalar el muestreo no aleatorio llevado a cabo, ya que todos los participantes fueron estudiantes universitarios, por lo que la mayoría de ellos tenían edades muy similares (alrededor de los 18 y 24 años). Esto pudo influir en los resultados obtenidos en cuanto a que la edad no resultó estar relacionada con los niveles de burnout en ninguna de sus dimensiones. A pesar de coincidir estos resultados con los de estudios previos, se sugiere como mejora para futuras investigaciones realizar un muestreo aleatorio.

Asimismo, otra posible mejora sería la construcción de un cuestionario ad hoc para registrar los datos necesarios en lugar del IPAQ, ya que este cuestionario está referido a los últimos 7 días (sería preferible preguntar acerca de la actividad física realizada normalmente, ya que sus respuestas podrían en algunos casos no ser representativas de lo que hacen generalmente).

Otra posible orientación para futuras investigaciones sería el registro de qué deporte o actividad física concreta realizan, ya que esta podría ser una variable influyente y podrían encontrarse diferencias significativas a partir de lo cual analizar qué variables influyen en que esos deportes estén relacionados con mayores niveles de burnout.

En definitiva, se espera que este estudio de pie a futuras investigaciones en esta línea, y en especial de cara a la intervención y prevención del síndrome una vez conocidas qué características de los deportistas están asociadas a un mayor riesgo de padecerlo.

\section{Referencias}

1. Alarcon, G., Eschleman, K y Bowling, N. (2009). Relationships between personality variables and burnout: A meta-analysis. Work \& Stress, 23(3), 244-263. https://doi.org/10.1080/02678370903282600

2. Aluja, A., Blanch, A., Sole, D., Dolcet, J. M., y Gallart, S. (2009). Versiones cortas del NEO-PI-R: el NEO-FFI frente al NEO-FFI-R. Behavioral Psychology/Psicología Conductual, 17(2), 335-351.

3. American Psychological Association (2015). The Road to Resilience. Recuperado de: http://www.apa.org/helpcenter/road-resilience. aspx

4. Arnold, R. y Fletcher, D. (2012). A research synthesis and taxonomic classification of the organizational stressors encountered by sport performers. Journal of Sport Exercise and Psychology, 34, 397-429. https:// doi.org/10.1123/jsep.34.3.397

5. Bermúdez, J. (1999). Personality and health protective behaviour. European Journal of Personality, 13, 83-103. https://doi. org/10.1002/(SICI)1099-0984(199903/04)13:2\%3C83::AIDPER338\%3E3.0.CO;2-M

6. Costa, P. y McCrae, R. (1992). Neo-PI-R: Revised NEO Personality Inventory (NEO-PI-R). Odessa, FL: Psychological Assessment Resources.

7. Costa, Py McCrae, R. (2008). The five-factor theory of personality. En O. John, R. Robins y L. Pervin (Eds.). Handbook of personality (3rded.) (pp.159-181). Nueva York: Guilford Press.

8. Currie, A. y Johnston, A. (2016). Psychiatric disorders: The psychiatrist's contribution to sport. International Review of Psychiatry, 28(6), 587-594. Doi: https://doi.org/10.1080/09540261.2016.1197188

9. De Francisco, C., Garcés de los Fayos, E. y Arce, C. (2013). Burnout en deportistas: Prevalencia del síndrome a través de dos medidas. Cuadernos de Psicología del Deporte, 14(1), 29-38.

10. De Francisco, C., Arce, C., Del Pilar Vílchez, M., y Vales, Á. (2016). Antecedentes y consecuencias del burnout en deportistas: estrés percibido y depresión. International Journal of Clinical and Health Psychology, 16(3), 239-246.

11. Edward, K.L. (2005). The phenomenon of resilience in crisis care mental health clinicians. International Journal of Mental Health Nursing, 14(2), 142-148. https://doi.org/10.1111/j.1440-0979.2005.00371.x

12. Freudenberger, H. J. (1974). Staff burnout. The Journal of Social Issues, 30(1), 159-166.

13. Garcés de los Fayos, E. y Cantón, E. (1995). El cese de la motivación: el Síndrome del Burnout en deportistas. Revista de Psicología del Deporte, 4(2), 151-160.

14. Garcés de los Fayos, E. (1999). Burnout en deportistas: Un estudio de la influencia de las variables de personalidad, sociodemográficas y deportivas en el sindrome. Universidad de Murcia: Tesis Doctoral no publicada.

15. Garcés de los Fayos, E. (2004). Burnout en deportistas. Madrid, España: Editorial EOS Instituto de orientación psicológica asociados.

16. Garcés de los Fayos, E., Carlín, F. y Carlín, M. (2010). Burnout en el 
contexto deportivo: análisis teórico-práctico del estado de la cuestión. Apuntes de Psicología, 28(2), 213-223.

17. Garcés de los Fayos, E., De Francisco, C. y Arce, C. (2012). Inventario de Burnout en Deportistas Revisado. Revista de Psicología del Deporte, 21(2), 271-278.

18. García-Parra, N., González, J. y Garcés de los Fayos, E. (2016). Estado actual del síndrome de burnout en el deporte. Cuadernos de Psicología del Deporte, 16(2), 21-28.

19. Gould, D., Tuffey, S., Udry, E. y Loehr, J. (1996). Burnout in competitive junior tennis players: I. A quantitative psychological assessment. The Sport Psychologist, 10(4), 322-340. https://doi.org/10.1123/tsp.10.4.322

20. Gustaffson, H., Kenttä, G., Hassmén, P. y Lundqvist, C. (2007). Prevalence of burnout in adolescent competitive athletes. The Sport Psychologist, 21, 21-37. https://doi.org/10.1123/tsp.21.1.21

21. Hill, A.P., Hall, H.K., Appleton, P.R., y Kozub, S.A. (2008). Perfectionism and burnout in junior elite soccer players: The mediating influence of unconditional self-acceptance. Psychology of Sport and Exercise, 9, 630-644. https://doi.org/10.1016/j.psychsport.2007.09.004

22. Hosseini, S.A. y Besharat, M.A. (2010). Relation of resilience whit sport achievement and mental health in a sample of athletes. Procedia Social and Behavioral Sciences, 5, 633-638. https://doi.org/10.1016/j.sbspro.2010.07.156

23. Howard, S., y Johnson, B. (2004). Resilient teachers: Resisting stress and burnout. Social Psychology of Education, 7(4), 399-420.

24. Jiménez, G., Jara, P. y García, C. (1995). Variables relevantes en el contexto deportivo y síndrome de burnout. V Congreso Nacional de Psicología de la Actividad Fisica y el Deporte. Valencia.

25. Kania, M.L., Meyer, B.B., y Ebersole, K.T. (2009). Personal and environmental characteristics predicting burnout among certified athletic trainers at national collegiate athletic association institutions. Journal of Athletic Training, 44(1), 58-66.

26. Maslach, C. y Jackson, S.E. (1981). MBI: Maslach Burnout Inventory. Manual. Palo Alto: University of California, Consulting Psychologists Press.

27. Maslach, C. y Jackson, S.E. (1986). MBI: Maslach Burnout Inventory. Manual Research Edition. Palo Alto: University of California, Consul- ting Psychologists Press.

28. Medina Mojena, G. (2001). Sindrome de Burnout y Locus de Control en deportistas cubanos de alto rendimiento. Tesis de Licenciatura no publicada. La Habana: Universidad de la Habana.

29. Reche, C., Tutte, V. y Ortín, F. J. (2014). Resiliencia, optimismo y burnout en judokas de competición uruguayos. Revista Iberoamericana de Psicología del Ejercicio y el Deporte, 9(2), 267-279.

30. Rew, L., Taylor-Seehafer, M., Thomas, N.Y. y Yockey, R.D. (2001). Correlates of resilience in homeless adolescents. Journal of Nursing Scholarship, 33, 33-40. https://doi.org/10.1111/j.1547-5069.2001.00033.x

31. Reynaga, P. y Pando, M. (2005). Relación del síndrome de agotamiento crónico (burnout), con el trastorno psicológico potencial en jóvenes deportistas. Investigación en Salud, 7, 153-160.

32. Reynaga, P. (2008). Presencia de trastorno psicológico y burnout en jóvenes deportistas jaliscienses de alto nivel. Presentado en el II Congreso de la Sociedad Iberoamericana de Psicología de la Actividad Física y del Deporte, Torrelavega, Cantabria.

33. Reynaga, P. (2009). Prevalencia del sindrome de agotamiento crónico ("burnout") en jóvenes deportistas de alto rendimiento de Jalisco. Presentado en el Primer Encuentro online de Psicología del Deporte de la SIPD, México.

34. Rotella, R.J., Hanson, T. y Coop, R.H. (1991). Burnout in youth sports. Elementary School Journal, 91(5), 421-428.

35. Ruiz-Barquín, R., de la Vega-Marcos, R., Poveda, J., Rosado, A., y Serpa, S. (2012). Análisis psicométrico de la Escala de Resiliencia en el deporte del fútbol. Revista de Psicología del Deporte, 21(1), 143-151.

36. Wagnild, G., y Young, H. (1993). Development and psychometric evaluation of the Resilience Scale. Journal of Nursing Measurement, 1(2), $165-178$.

37. Weinberg, K.S. y Gould, R. (1995). Foundations of sport and exercise psychology. Champaigns, Illinois: Human Kinetics

38. WHO/OMS. Recomendaciones Mundiales sobre Actividad Física para la Salud. Ginebra: Organización Mundial de la Salud; 2010.

39. Yi, J., Smith, R. y Vitaliano, P. (2005). Stress-resiliencie, illness and coping: a person-focused investigation of young women athletes. Journal of Behavioral Medicine, 28(3), 257-265. 